\title{
Real Characterization on Order Banach Algebra
}

\author{
Boushra Youssif \\ Department of Mathematics,Al-QadisiyaUniversity, Iraq \\ Boushra.alshebani@qu.edu.iq \\ Sarah Fahim Hassan \\ Department of Mathematics, Al-Qadisiya University, Iraq \\ Sarahfahim66@yahoo.com
}

\begin{abstract}
In this paper we will study the real character on order Banach algebra with identity, and study character onorder Banach algebrawithout identity. We proved some properties on real character it. We define set of all character and prove it with some condition convex set and used it to prove all character is extreme point, we introduce dual cone in order Banach space .Also we show any order Banach algebra satisfying certain conditions is isomorphic to the space valued continuous function $\mathrm{C}_{0}(\mathrm{x})$ forsuitable a locally compact Housdorf space.
\end{abstract}

Keywords: order Banach algebra; algebra cone; dual cone; character.

\section{Introduction}

The study of Banach algebra entails combining the use of algebraic and analytic (or topological) methods. It has become an important field modern operator theory having many practical and theoretical applications.

We will show that Banach algebra techniques combine with order structures yield new insights. We prove our results in structure will called order Banach algebra $A$, the ordering which is induced by a subset $C$ of $A$ with certain special properties, called an algebra cone which is compatible with the algebraic structure of $A$, and this the useful references for the material on Banach space are [J.B.Conway,1990].References for Banach algebra are [D,Holland,2015], [J.M.Erdman,2011] and references for order Banach Algebra are[D.Robimsond,1983],[H.Rouben,1996], and [R.Dejong,2010].

1-order Banach algebra

Definition 1.1[1 ] :- Let $A$ be a Banach with respect to real numbers. Ais called Branch algebra, if there exist operation from $A \times A$ to $A$ such that $(x, y) \rightarrow x y$. for all $x, y$ and $z$ belong to $A$ and $\alpha \in \mathbb{R}$ with the following properties

$$
\begin{aligned}
& 1-(x y) z=x(y z) \\
& 2-(\lambda x+y) z=\lambda x z+y z a n d z(\lambda x+y)=\lambda z x+z y \text { (commutive) } \\
& \text { 3- }\|x y\| \leq\|x\|\|y\| \text { (Sub multiplicative) }
\end{aligned}
$$

Definition 1.2[1]:-We called a Banach algebra $A$ is until, if it has a unite element e such that e. $x=x . e=x \forall x \in A$. If $\mathrm{A}$ has unite then $\|\mathrm{e}\|=1$

Definition ( $\mathbf{W}^{*}$-topology) 1.3[5]:-Let $A$ be a Banach space and $A^{*}$ be the dual space of $A$, the weak topology is the weakest topology such the map $T: A \rightarrow R$ is continuous .A weak*topology is the weakest topology making all functional on $A^{*}$ are continuous.

Theorem (Hahn Banach) 1.4[6]:- Let $X$ be a linear space in a real numbers $\mathbb{R}$ and let $q$ be a sub linear functional on $X$, if $M$ be a linear subset of $X$ and if $f: M \rightarrow \mathbb{R}$ be a linear functional with $f(x) \leq q(x)$ for all $x \in M$. Then there exist a linear functionalK $: X \rightarrow \mathbb{R}$ with $\mathrm{K}(\mathrm{x}) \leq \mathrm{q}(\mathrm{x})$ for all $\mathrm{x} \in \mathrm{X}$.

Proposition 1.5[6]:-If $X$ is a normal space and $\mathrm{x}$ in $\mathrm{X}$. Then $\|\mathrm{x}\|=\sup \{|\mathrm{f}(\mathrm{x})|: \mathrm{f} \in \mathrm{X} *:\|\mathrm{f}\| \leq 1\}$.

Definition1.6 [6]:-The closed unit ball in $X$ which denoted by $C L(x)$ when $X$ is normed space is define by $C L(x)=\{x \in X:\|x\| \leq$ $1\}$.

Theorem(Alaoglu's theorem) 1.7[4 ]:- $C L(x)$ is a $\mathrm{W}^{*}$-compact if $\mathrm{X}$ is normed space. 
Definition(Extreme point) 1.8[6 ]:-If $X$ is a linear space and $\mathrm{K} \subseteq \mathrm{X}$, such $\mathrm{K}$ is convex and $\mathrm{x} \in \mathrm{K}$, then $\mathrm{x}$ is extreme point if there is no proper open line segment in $\mathrm{K}$, i.e if $x=t a+(1-t) b$, such that $\mathrm{a}, \mathrm{b} \epsilon \mathrm{K}$ and $0<t<1$ then $a=b$. And we denote to the set of extreme point by $e(K)$

Theorem (The KrienMilman theorem) 1.9[4]:-Let $X$ be a locally convex space andK bea non-empty compact convex subset of $\mathrm{X}$. Then $e(K) \neq \phi$ and is equal to theclosed convex hull of $e(K)$.

Definition 1.10[3]:- Let $A$ be a real Banach algebra with unit $e$ and $C$ non-empty subset of $A$.We call $C$ a cone if it satisfies the following

1. $a+b \in C$ for all $a, b \in C$,

2. $\lambda \mathrm{a} \epsilon \mathrm{C}$ for all $\mathrm{a} \epsilon \mathrm{C}$ and $\lambda \geq 0$.

In addition if $C$ satisfies $C \cap-C=\{0\}$, then $C$ will be called a proper cone induced an ordering $(\leq)$ on $A$ by $a \leq b$ if and only if $\mathrm{b}-\mathrm{a} \epsilon \mathrm{C}$ for all $\mathrm{a}, \mathrm{b} \in \mathrm{C}$.We say that $\mathrm{C}$ is algebra cone if it is satisfies the following:

1. $a \cdot b \in C$ for all $a, b \in C$,

2. $\mathrm{e} \epsilon \mathrm{C}$.

Definition (Ordered Banach Algebras) 1.11[3]:- Let A be a real Banach algebra with unit e is called order Banach algebra (OBA) when $\mathrm{A}$ is ordered by a relation $(\succcurlyeq)$ such that for every a,b,c $\epsilon \mathrm{C}$ and $\lambda \geq 0$
1. $a, b \geqslant 0 \Rightarrow a+b \geqslant 0$
2. $a \geqslant 0, \lambda \geq 0 \Rightarrow \lambda a \geqslant 0$
3. $\mathrm{a}, \mathrm{b} \geqslant 0 \Rightarrow \mathrm{a} \cdot b \geqslant 0$.
4. $\mathrm{e} \geqslant 0$

So if $A$ ordered by an algebra cone $C$, we will obtain $(A, C)$ is an order Banach algebra.

Definition 1.12[6 ]:- Let $A$ be $O B A$ and $C$ be algebra cone so

- We will say that $\mathrm{C}$ is normal if there is $\beta>0$, and for any $\mathrm{a}, \mathrm{b} \in \mathrm{A}, 0 \leq a \leq b \Rightarrow\|\mathrm{a}\| \leq \beta\|\mathrm{b}\|$.

- We will say that $\mathrm{C}$ is $\alpha$-normal if there is $\alpha>0$, and for any $\mathrm{a}, \mathrm{b}, \mathrm{c} \in \mathrm{A}, \mathrm{b} \leq a \leq c \Rightarrow\|\mathrm{a}\| \leq \alpha$ maxi( $\|\mathrm{b}\|$, , ill $\mathrm{c} \|)$.

If $\alpha=1$ we say that $\mathrm{C}$ is $1-\max$-normal.

Proposition 1.13[6]:-Every normal algebra cone $\mathrm{C}$ is a proper algebra cone.

Definition 1.14:- Let A be aorder Banachalgebra, we called the function $f$ ismultiplicative if satisfies: $f(a b)=f(a) f(b)$.A functional $f$ from order Banach $A$ into the set of real numbers $R$ is called real character if $f$ is linear, multiplicative, and $f(e)=1$

. Notation:-When $A$ is ordered by an algebra cone $C$. If we denote $\mathrm{M}_{\mathrm{A}}$ to the set of all real characters, and $\mathrm{M}_{\mathrm{A}}{ }^{+}$to the subset of $\mathrm{M}_{\mathrm{A}}$ such that $\mathrm{f}(\mathrm{x}) \geq 0$,for all $\mathrm{x}$ in $\mathrm{C}$.

Lemma 1.15: $-M_{A}$ and $M_{A}{ }^{+}$are convex sets.

Proof: - Let $f_{1}, f_{2} \in M_{A}$ and $0 \leq \beta \leq 1, a, b \in A$

$$
\begin{aligned}
1-\left(\beta f_{1}+(1-\beta) f_{2}\right)(a+b)=\beta f_{1}(a & +b)+(1-\beta) f_{2}(a+b) \\
& =\beta f_{1}(a)+\beta f_{1}(b)+(1-\beta) f_{2}(a)+(1-\beta) f_{2}(b) \\
= & \beta f_{1}(a)+(1-\beta) f_{2}(a)+\beta f_{1}(b)++(1-\beta) f_{2}(b) \\
& =\left(\beta f_{1}+(1-\beta) f_{2}\right)(a)+\left(\beta f_{1}+(1-\beta) f_{2}\right)(b)
\end{aligned}
$$

$2-\left(\beta f_{1}+(1-\beta) f_{2}\right)(\alpha a)=\beta f_{1}(\alpha a)+(1-\beta) f_{2}(\alpha a)$

Since $f_{1}, f_{2} \in M_{A}$ then we obtain 


$$
=\alpha \beta f_{1}(a)+\alpha(1-\beta) f_{2}(a)=\alpha\left[\beta f_{1}(a)+(1-\beta) f_{2}(a)\right] \quad=\alpha\left[\beta f_{1}+(1-\beta) f_{2}\right](a)
$$

3-To prove that $\left[\beta f_{1}+(1-\beta) f_{2}\right](a b)=\left(\beta f_{1}+(1-\beta) f_{2}\right)(a) \cdot\left(\beta f_{1}+(1-\beta) f_{2}\right)(b)$

Let $0 \leq \beta \leq 1, \varepsilon>0$ and $f_{1,} f_{2} \in M_{A}$

Lett $=\frac{i}{2^{n}}$ for some $i, n \in \mathbb{N}$, such that $|\beta-t|<\varepsilon$

And $\mathrm{t} f_{1}+(1-t) f_{2} \in \mathrm{M}_{\mathrm{A}}$.

Let $\left\{f_{\lambda}\right\}_{\lambda \in \Lambda}$ be net in $\mathrm{M}_{\mathrm{A}}$ satisfy $f_{\lambda} \rightarrow \beta, \lambda \in \Lambda$ and

$\left.f_{\lambda} f_{1}(a b)+\left(1-f_{\lambda}\right) f_{2}(a b)=f_{\lambda} f_{1}+\left(1-f_{\lambda}\right) f_{2}\right)(\mathrm{a}) \cdot\left(f_{\lambda} f_{1}+\left(1-f_{\lambda}\right) f_{2}\right)(\mathrm{b})$ for every a,beA

Hence $\left.\left(\beta f_{1}(\mathrm{ab})+(1-\beta) f_{2}(\mathrm{ab})\right)=\beta f_{1}+(1-\beta) f_{2}\right)(\mathrm{a}) \cdot\left(\beta f_{1}+(1-\beta) f_{2}\right)(\mathrm{b})$, for every $\mathrm{a}, \mathrm{b} \in \mathrm{A}$.Then from (1) ,(2) ,(3) we obtain $\beta f_{1}+(1-\beta) f_{2} \in$ MA.

$M_{A}{ }^{+}$Will be convex by follows the covexity of $\mathrm{M}_{\mathrm{A} . \square}$

Proposition 1.16:- Let A be anOBA without identity and $f$ be linear, multiplicative on $\mathrm{A}$. Then we can extended $f$ to real character $\bar{f}$ on OBA with identity $A_{e}$ such that Ais sub algebra of $A_{e}$ and $\bar{f} /{ }_{A}=f$.

Proof:- Let $\mathrm{A}$ be a OBA, and let $A_{e}=A \times \mathbb{R}$

To prove that $A_{e}$ is an OBA with identity asfollows :

\section{a- Algebra}

We, define operations on, $A_{e}$ as follows:

$1-(a, \alpha)+(b, \mu)=(a+b, \alpha+\mu) \quad$ for all $a, b \in A, \alpha, \mu \in \mathbb{R}$

2- $\beta(a, \mu)=(\beta a, \beta \mu)$ for all $\beta \in R, a \in A$

$3-(a, \alpha) \cdot(b, \mu)=(a b+\alpha b+\mu a, \alpha \mu)$.

It clear that $A_{e}$ is an algebra with identity $(0,1)$ and $\mathrm{A}$ is sub algebra of $A_{e}$.

\section{b- Normed algebra}

If $\|(a, \alpha)\|=\|\mathrm{a}\|=|\alpha|$ for all $\mathrm{a} \in A, \alpha \in \mathbb{R}$. Then $A_{e}$ be a normed algebra.

\section{c- Banach algebra}

To prove that $A_{e}$ be a complete.

Let $\left\{x_{n}, \alpha_{n}\right\}$ be a Cauchy sequence in $A_{e}$

for all $\varepsilon>0$, there exist $k \in \mathbb{Z}^{+}$such that $\left\|\left(x_{n}, \alpha_{n}\right)-\left(x_{m}, \alpha_{m}\right)\right\|<\varepsilon \forall \mathrm{n}>\mathrm{k}$

$\left\|\left(x_{n}-x_{m}, \alpha_{n}-\alpha_{m}\right)\right\|<\varepsilon \forall \mathrm{n}>\mathrm{k}$

$\left\|x_{n}-x_{m}\right\|+\left|\alpha_{n}-\alpha_{m}\right|<\varepsilon$

$\left\|x_{n}-x_{m}\right\|<\varepsilon$ and $\left|\alpha_{n}-\alpha_{m}\right|<\varepsilon$

Since $\left\{x_{n}\right\}$ be a Cauchy sequence in $A,\left\{\alpha_{n}\right\}$ be a Cauchy sequence in $\mathbb{R}$. since $\mathrm{A}$ is Banach algebra ,there exist $x \in A$ such that $x_{n} \rightarrow x$ as $n \rightarrow \infty$

i.e $\left\|x_{n}-x\right\|<\varepsilon$ for all $n \geq k$

Since $\mathbb{R}$ is complete so there exist $\alpha \in \mathbb{R}$ such that $\left|\alpha_{n}-\alpha\right|<\varepsilon$

Obtain $\left\|x_{n}-x\right\|+\left|\alpha_{n}-\alpha\right|<\varepsilon \varepsilon$ for all $n \geq k$

Implies that $\left\|\left(x_{n}, \alpha_{n}\right)-\left(x_{m}, \alpha_{m}\right)\right\|<\varepsilon \varepsilon$ for all $n \geq k$

Since $\left\{x_{n}\right\}$ in $\mathrm{A}$ and, $\left\{\alpha_{n}\right\}$ in $\mathbb{R}$. 
Then $\left\{\left(x_{n}, \alpha_{n}\right)\right\}$ in $A_{e}$ so $A_{e}$ is complete .

\section{d- Order Banach algebra}

Let $\mathrm{a}, \mathrm{b} \in A, a \geqslant 0, b \geqslant 0$ since $\mathrm{A}$ is OBA then $\mathrm{a}+\mathrm{b} \geqslant 0$ and if $\alpha \geq 0, \mu \geq 0$ then $\alpha+\mu \geq 0$.

Now to show that $C_{e}=C \times \mathbb{R}$ be an algebra cone, let $a, b \in C$ and $\alpha, \mu \in \mathbb{R}$ such that $x=(a, \alpha), y=(b, \mu) \epsilon C_{e}$.

1) $x+y=(a, \alpha)+(b, \mu)=(a+b, \alpha+\mu) \epsilon C_{e} \quad$ for all $x, y \epsilon C_{e}$,

2) $\mu x=\mu(a, \alpha)=(\mu a, \mu \alpha) \epsilon C_{e}$,

3) $x \cdot y=(a, \alpha) \cdot(b, \mu)=(a b+\alpha b+\mu a, \alpha \mu)$.

4) $(0,1) \in C_{e}$

A cone $C_{e}$ on $A_{e}$ induced an ordering relation $\geqslant$ on $A_{e}$ satisfies:

1-[reflaxive]

for all $(a, \alpha) \in A_{e}$ since $a \geqslant a$ and $\alpha \geq \alpha$.Then $(a, \alpha) \geqslant(a, \alpha)$

2-[transitive]

For all $(a, \alpha),(b, \mu),(c, \lambda) \in A_{e}$ since $a \geqslant b, b \geqslant c$ obtain $a \geqslant c$, and since $\alpha \geq \mu, \mu \geq \lambda$

obtain $\alpha \geq \lambda$ so if $\quad(a, \alpha) \geqslant(b, \mu)$ and $(b, \mu) \geqslant(c, \lambda)$ then $(a, \alpha) \geqslant(c, \lambda)$

Now to satisfy conditions of OBAfor all $(a, \alpha),(b, \mu) \in A_{e},(a, \alpha) \geqslant(b, \mu)$ such that $a \geqslant b \alpha \geq \mu$

$$
\begin{aligned}
& 1-(a, \alpha) \geqslant 0,(b, \mu) \geqslant o \Rightarrow(a+b, \alpha+\mu) \geqslant 0 \\
& 2-(a, \alpha) \geqslant 0, \lambda \geq 0 \quad \Rightarrow \lambda(a, \alpha)=(\lambda a, \lambda \alpha) \geqslant 0 \\
& 3-(a, \alpha) \geqslant 0,(b, \mu) \geqslant 0 \Rightarrow(a, \alpha) .(b, \mu)=(a b+\alpha b+\mu a, \alpha \mu) \geqslant 0, \\
& 4-(0,1) \geqslant 0 \text {. }
\end{aligned}
$$

\section{e- $\bar{f}$ character}

We

$$
\text { define } \bar{f}: A_{e} \rightarrow \mathbb{R} \text { asfollows: } \bar{f}((a, \alpha))=f(a)+\alpha
$$

foralla $a A, \alpha \in \mathbb{R}$

To prove that $\bar{f}$ is character

$$
\begin{array}{lc}
\bar{f}((a, \beta)+(b, \alpha))=f(a+b, \beta+\alpha)=f(a)+f(b)+\alpha+\beta=\bar{f}(a, \alpha)+\bar{f}(b, \alpha) \\
\bar{f}((\lambda a, \lambda \alpha)=f(\lambda a)+\lambda \alpha=\lambda \bar{f}(a, \alpha) \\
\bar{f}((a, \alpha)(b, \mu))=\bar{f}(a b+\alpha b+\mu a . \alpha \mu) & \\
& =f(a b+\alpha b+\mu \alpha)+\alpha \mu \\
& =f(a b)+f(\alpha b)+f(\mu \alpha)+\alpha \mu \\
=\bar{f}((a, \alpha)) \cdot \bar{f}((b, \mu)) \text { for all } \mu \in \mathbb{R} & =[f(a)+\alpha] .[f(b)+\mu]
\end{array}
$$

Hence we obtain that $\bar{f}$ is realcharacter.

Definition1.19:- $A_{e}$ is called order adjunction with identity of an order Banach algebra without identity $(\mathrm{A}, \mathrm{C})$.

Proposition 1.20 [1]:- Let $A$ be a real Banach algebra with identity and $\mathrm{a} \epsilon A$ is an invertible element,suppose $\mathrm{b} \epsilon A$ with $\|b-a\|<$ $\frac{1}{\left\|a^{-1}\right\|}$, then $b$ is invertible and $\left\|(a-b)^{-1}\right\| \leq \frac{\left\|a^{-1}\right\|^{2}\|b-a\|}{1-\left\|a^{-1}\right\|\|b-a\|}$.

Lemma1.21:- let $A$ be a real Banach algebra without identity. Then for every character in $M_{A}$ is bounded. 
Proof: - Since A without identity then we extend $f$ to the character $\bar{f}$ of $A_{e}=A \times \mathbb{R}$ in this way we have foi such that $i: A \rightarrow A_{e}$ defined by $(\mathrm{a})=(\mathrm{a} . \mathrm{o})$, which is inclusion map.Toprove thatfis bounded i.e.to prove $|\mathrm{f}(\mathrm{a})| \leq\|\mathrm{a}\|$

Assume that $a \in A_{e}$ with $|\mathrm{f}(\mathrm{a})|>\|\mathrm{a}\|$.

Take $\beta=\mathrm{f}(\mathrm{b})$ and $\mathrm{a}_{1}=\beta^{-1} \mathrm{~b}$ which has a norm $\left\|\mathrm{a}_{1}\right\|<1$ by proposition (1.20)a $=1$ and $\mathrm{b}=1-\mathrm{a}_{1}$, which follows that $1-\mathrm{a}_{1}=1-$ $\beta^{-1} \mathrm{~b}$ is invertible,

since $\beta \neq 0$, so we have $c=\beta\left(1-a_{1}\right)=\beta 1-$ bis invertible,

weobtainf $(c)=\beta f(1)-f(b)=\beta-f(b)=0$,

but

$1=f(1)=f\left(c c^{-1}\right)=f\left(c^{-1}\right) f(c)=0$ which is imposible, so $|f(a)| \leq\|a\| . \quad \square$

\section{Dual cone in Order Banach algebra}

Definition 2.1[6 ]:- Let $A^{*}$ be a dual of order Banach space with algebra cone $\mathrm{C}$, a dual cone is the set of all continuous (bounded) linear functional and it is non-negative on $C$ which isdenoted by $C^{*}$ i.e $C^{*}=\left\{f \epsilon A^{*}: f(x) \geq 0\right.$, for all $\left.x \in C\right\}$

Lemma 2.2[ 6]:- Let $S$ be a closed and convex set of aBanach space A with respect to real numbers and a $\notin S$.Then there is a continuous (bounded) linear functional $f$ such that $f(a)<f(b)$ for all $\mathrm{b} \in C$.

Lemma 2.3:- Let $C$ be an algebra cone in A.Then $a \epsilon C$ if and only if $f(a) \geq 0$ for all $f \epsilon C^{*}$ and $C \neq A$ implies $C^{*} \neq\{0\}$

Proof:- Suppose that $a \notin C$, since $\mathrm{C}$ is closed and convex cone so from above lemmawith $f(a)<f(b)$ forall $b \epsilon C$, thus $f(a)<0=f(0)$, since $\mathrm{b} \epsilon C$ then we obtain $\beta b \epsilon C$ and $\beta>0$. Then $f(b) \geq 0$, Hence $f \in C^{*}$.

Definition2.4]:- Let $A^{*}$ be a dual of order Banach space and $C^{*}$ be a dual cone in $A^{*}$ which is called - generated , if for each $f \in A^{*}$ and $g_{1}, g_{2} \in C^{*}$ such that $f=g_{1-} g_{2}$ satisfies that $\alpha\|f\| \geq\left\|g_{1}\right\|+\left\|g_{2}\right\|$, If $f=g_{1-} g_{2}$ for each $g_{1}, g_{2} \epsilon C^{*}$ and $\|f\|=$ $\left\|g_{1}\right\|+\left\|g_{2}\right\|$.

we will call that $C^{*}$ by 1 -generated.

Proposition 2.5[ 2]:- Let $(A, C)$ be an order Banach algebra .Then $C$ is $\alpha$ - normal if and only if $C^{*}$ is $\alpha-$ generated .In particular Cis $1-$ normalif and only if eachf $\in A^{*}$ satisfies $f=g_{1-} g_{2}$ for each $g_{1}, g_{2} \in C^{*}$.

Definition 2.8:- A closed ball of $A^{*}$ which is denoted by $\widetilde{J}_{A}=\left\{f \in A^{*}:\|f\| \leq 1\right\}$.

Lemma2.9:- $M_{A}$ and $M_{A}^{+}$and $\Im_{A}^{+}=\mathfrak{I}_{A}+C^{*}$ are $W^{*}$ - compact

Proof: -Since $\|f\| \leq 1$ and $M_{A} \subset \mathfrak{I}_{A}$ then we obtain $\mathfrak{J}_{A}$ is $W^{*}$-compact

since $A^{*}$ is normed space. Then $\widetilde{\Im}_{A}=C L\left(A^{*}\right)$ by [Alaoglu'stheorem].

To show that $M_{A}$ is $W^{*}$-compact we have to prove $M_{A}$ is $W^{*}$-closed in $\mathfrak{J}_{A}$

Let take a net $\left(f_{\lambda}\right)_{\lambda \in \Lambda}$ in $M_{A}$ and $f \in \mathfrak{J}_{A}$ such that $f=\lim _{\lambda \in \Lambda} f_{\lambda}$ to show that $f \in M_{A}$

$$
f(a b)=\lim _{\lambda \in \Lambda} f_{\lambda}(a b), \forall a, b \in A
$$

It is clear from that $f$ is liner and Multiplicative and $f$ is not trivial since $f(e)=\lim _{\lambda \in \Lambda} f_{\lambda}(e)=1$

Hence $f \in M_{A}$ and $M_{A}$ is $W^{*}$ - closed in $\mathfrak{J}_{A}$.

To show that $M_{A}{ }^{+}$is $W^{*}$ - compact we have to prove that $M_{A}{ }^{+}$is $W^{*}$-closed

Let $\left(f_{\lambda}\right)_{\lambda \in \Lambda}$ be a net in $M_{A}{ }^{+}$and let $f \in M_{A}{ }^{+}$

Then for $a \in C$ we get $\left(f_{\lambda}\right)(a) \geq 0$ so $f(a) \geq 0$ for all $a \in C$

Thus $f \in M_{A}{ }^{+}$and $M_{A}{ }^{+}$is $W^{*}$-closed 
To prove that $\mathfrak{J}_{A}^{+}$is $W^{*}$-closed

Let $\left(f_{\lambda}\right)_{\lambda \in \Lambda}$ be a net in $\mathfrak{J}_{A}^{+}$and let $\|f\| \leq 1$, such that $f \in M_{A}$ and $f=\lim _{\lambda \in \Lambda} f_{\lambda}$.

Hence therefor $a \in C$ we have $\left\|f_{\lambda}(a)\right\| \geq 0$ and $\|f(a)\| \geq 0$

Thus $f \in \mathfrak{I}_{A}^{+}$and we obtain $\mathfrak{\Im}_{A}^{+}$is $W^{*}$-closed.

\section{Main results}

Proposition3.1:- every character in $M_{A}$ is extreme point.

Proof: - Let $f$ be a character and let $f=t f_{1}+(1-t) f_{2}$ such that $f_{1}, f_{2} \in M_{A}$

To prove that $f_{1}=f_{2}$

Assume that $f_{1} \neq f_{2}$, there exist $a \in A$ such that $f_{1}(a) \neq f_{2}(b)$. Then $t f_{1}(a) \neq t f_{2}(b)$ and $(1-t) f_{1}(a) \neq(1-t) f_{2}(b)$,implies that $t f_{1}(a) \cdot f_{1}(b)+(1-t) f_{1}(a) f_{1}(b) \neq t f_{2}(a) \cdot f_{2}(b)+(1-t) f_{2}(a) \cdot f_{2}(b)$ for someb $\neq 0$.

$t f_{1}(a) \cdot f_{1}(b)+(1-t) f_{2}(a) \cdot f_{2}(b) \neq\left(t f_{1}(a)+(1-t) f_{2}(b)\right) \cdot\left(t f_{1}(b)+(1-t) f_{2}(b)\right)$

Then $f(a b) \neq f(a) f(b)$ this impossible because $f$ character.

- $\quad$ Now we consider the following conditions

1- $\bar{f}(y a b) \geq 0$ for every $f \in M_{A}^{+}$and $a, b, y \in A_{e}$

2- $(y a) \in C_{e}$ for every $a \in C_{e}$, and $y \in A_{e}$.

Lemma 3.2:- let $A$ be real order Banach algebraand $\left\{f_{\lambda}\right\}_{\lambda \in \Lambda}$ be a net $A_{e}$ satisfying that $f_{\lambda} a \rightarrow a$ for all $a \in A_{e}$, let $y \in$ $A_{e}$.then $A_{e}$ satisfies the conditions (1) and (2).

Proof: - Let $\left\{f_{\lambda}\right\}_{\lambda \in \wedge}$ a net and $f_{\lambda} \mathrm{a} \rightarrow \mathrm{a}$ for all $\mathrm{a} \in A_{e}$, let $y \in A_{e}$, then we have $y f_{\lambda} \in C_{e}$ and $\left(a b f_{\lambda}\right) \in C_{e}$ so $\left(y f_{\lambda}\right)\left(a b f_{\lambda}\right) \in C_{e}$

Then $\left(y f_{\lambda}\right)\left(a b f_{\lambda}\right)$ is positive, thus $\left(y f_{\lambda}\right)\left(a b f_{\lambda}\right) \geq 0$

Let $y^{\prime}, a^{\prime}, b^{\prime} \in A$ and $\alpha, \beta \in \mathbb{R}$ such that $y=\left(y^{\prime}, \beta\right), a b=\left(\left(a^{\prime} b^{\prime}\right), \alpha\right)$

Without products $\left(y f_{\lambda}\right)\left(a b f_{\lambda}\right)$ and $y a b$, since $f$ is continuous and liner map we obtain $f\left(\left(y f_{\lambda}\right)\left(a b f_{\lambda}\right)-\alpha \beta f_{\lambda}\right)$

Convergent to $\bar{f}\left(\left(f_{\lambda}\right)\left(a b f_{\lambda}\right)-\alpha \beta\right)$, since $\|f\| \leq 1$

We have $f\left(\beta \alpha f_{\lambda}\right) \leq \beta \alpha$

$\left.\left.0 \leq f\left(y f_{\lambda}\right)\left(a b f_{\lambda}\right)=\mathrm{f}\left(\mathrm{y} f_{\lambda}\right)(a b)-\beta \alpha f_{\lambda}\right)+f\left(\beta \alpha f_{\lambda}\right) \leq f\left(y f_{\lambda}\right)\left(a b f_{\lambda}\right)-\beta \alpha f_{\lambda}\right)+\beta \alpha \quad$ Convergent to $\bar{f}(y a b-\beta \alpha)+\beta \alpha=\bar{f}(y a b)$

so condition (1) is satisfied and condition (2)directly by a $\lim _{\lambda \in \Lambda}\left(y f_{\lambda}\right) a . \square$

Proposition3.3:-let $X$ be a locally compact Hausdorff spaceand $C_{0}(X)=\{$ f:f: $X \rightarrow \mathbb{R}$, continuos $\}$,let $C_{0}^{+}(X)=\left\{f \in C_{0}(X): f(x) \geq\right.$ $0, \forall x \in X$, then $C O+X$ be an algebra cone and make $C O X$ by an OBA by $C O+X$.

Proof:-To show that $C_{0}^{+}(X)$ be an algebra cone ,let $f_{1}, f_{2} \in C_{0}^{+}(X), \lambda \geq 0$ and $x \in X$ such that $f_{1}(x) \geq 0, f_{2}(x) \geq 0$

$$
\begin{array}{ll}
\text { 1- } & f_{1}(x)+f_{2}(x)=\left(f_{1}+f_{2}\right)(x) \geq 0 \\
\text { 2- } & \lambda f_{1}(x) \geq 0 \quad \text { for all } \lambda \geq 0 \\
\text { 3- } & f_{1} f_{2}(x)=f_{1}(x) f_{2}(x) \geq 0 \\
\text { 4- } & f_{1}(1)=1 \geq 0 .
\end{array}
$$

It can show for every $f_{1}, f_{2}, f_{3} \in C_{0}^{+}(X)$ this satisfies that ordering is

$$
\begin{aligned}
& \text { a-[reflexive] i.e } f_{1} \geqslant f_{1} \text { if } f_{1}(x) \geq f_{1}(x) \text {. } \\
& \text { b-[transitive] i.e } f_{1} \geqslant f_{2} \text { and } f_{1} \geqslant f_{2} \text { then } f_{1} \geqslant f_{3} \text { if } f_{1}(x) \geq f_{2}(x) \text { and } f_{2}(x) \geq f_{3}(x) \text { then } f_{1}(x) \geq f_{3}(x) \text {. }
\end{aligned}
$$

So we obtain that $C_{0}^{+}(X)=\left\{f \epsilon C_{0}(X): f(x) \geq 0, \forall x \in X\right\}$ be an algebra cone which is make $C_{0}(X)$ an order Banach algebra(OBA). $\square$ 
Theorem3.4:- Let (A, C) be an order Banach algebra andC be a closed and $\alpha$ - normal cone. Then $\mathrm{A}$ isomorphic to $C_{0}(X)$,if and only if for a in A there is a net $\left\{f_{\lambda}\right\}_{\lambda \in \Lambda}$ such that $f_{\lambda} a \rightarrow$ a.

Proof:- let $F$ be a set of extreme points of $M_{A}$.

By (Urysohn'sLemma) that there is a net $\left\{f_{\lambda}\right\}_{\lambda \in \wedge}$ such that $f_{\lambda} a \rightarrow a$ satisfying for all a $\epsilon A$.

Now let $X=F /\{0\}$ with $w^{*}-$ topology .

Suppose that $\mathrm{C}=\mathrm{A}$.then for all $a, b \in A$ have $\leq a \leq b$.

Since $\mathrm{C}$ is $\alpha-$ normal this a contradiction,

Thus $C \neq A$ and $C^{*} \neq\{0\}$,

so by lemma $2.3 M_{A}^{+} \neq\{0\}$, thus $X \neq \emptyset$,

So $X=F /\{0\}$ is a locally compact Hausdorff space.

Let $\gamma: C(X) \rightarrow A$ defined by $\gamma(f)=f(a)$ for all $f \epsilon X$.

Since $f$ is character, so $\gamma$ is an algebra homomorphism.

And $\gamma$ will be isomorphism to show that, let $a, b \epsilon A$ such that $a \leq b$,so $\gamma(f)(b-a)=f(b-a) \geq 0$ for all $f \epsilon X$ and $\gamma(f)(b)-$ $\gamma(f)(a) \epsilon C^{+}(X, \mathbb{R})$,

$$
\text { thus } \gamma(f)(b) \geq \gamma(f)(a) .
$$

Conversely, let $a, b \in A$ and $\quad \gamma(f)(b) \geq \gamma(f)(a)$.

Then $f(b-a) \geq 0$, for all $f \in C(X)$,

so by(Klein- Mailman theorem) and since $M_{A}^{+}=F$ is the $w^{*}-$ closed convex hull and $M_{A}^{+}$generates $C^{*}$,

sof $(b-a) \geq 0$ for all $f \epsilon C^{*}$ and $b-a \epsilon C$ that by lemma 2.3.

Lemma3.5:- Let $(\mathrm{A}, \mathrm{C})$ be a real order Banach algebra with closed andnormal $\mathrm{C}$. Then $\mathrm{A}$ is an isomrphisim toC $\mathrm{C}_{0}(\mathrm{X})$ if and only if the following conditions are satisfies:-

1. Fora, $b \in A a n d\|a\|=\|b\|=\alpha$, there existc $\epsilon$ Awith $\|c\|=\alpha$ such that $c \geq a, b$ and $c \geq 0$

2.For $0 \leq a, b \leq 1, \alpha \geq 0$ and $\|a\|=\|b\|=\alpha$ Then $a b \leq a$ and $a b \leq b$

3.For every $\mathrm{x} \in \mathrm{C}$ there is two nets $\left\{\mathrm{f}_{\lambda 1}\right\}_{\lambda \in \Lambda},\left\{\mathrm{f}_{\lambda 2}\right\}_{\lambda \in \Lambda}$ of psitive elements and $\left\|\mathrm{f}_{\lambda 1}\right\| \leq 1,\left\|\mathrm{f}_{\lambda 2}\right\| \leq 1$ such that $\lim _{\mathrm{n}} \mathrm{ff}_{\lambda 1}=\mathrm{f}=$ $\lim _{\mathrm{n}} \mathrm{f}_{\lambda 2} \mathrm{f}$, let $\mathrm{C}$ be an algebra cone. Then there exist a net $\left\{\mathrm{f}_{\lambda}\right\}_{\lambda \in \Lambda}$ such that $\mathrm{f}_{\lambda} \mathrm{a} \rightarrow \mathrm{a}$ satisfies for all $\mathrm{a} \in \mathrm{A}$.

Proof:- To prove that $C_{0}(X)$ satisfies condition (1),(2) and (3)

let $f_{1}, f_{2} \in C_{0}(X)$ and $\left\|f_{1}\right\|=\left\|f_{2}\right\|=\alpha$, let $\left.f_{3}=\operatorname{maxi}\left|f_{1}\right|\left|f_{2}\right|\right\}$

then $f_{3} \in C_{0}(X)$ and $\left\|f_{3}\right\|=\alpha$ with $f_{3} \geq f_{1}, f_{2}$ and $f_{3} \geq 0$, so condition (1) satisfies.

If $f_{1}, f_{2} \in C_{0}^{+}(X)$ i.e $f_{1}(x) \geq 0$ and $f_{2}(\mathrm{x}) \geq 0$ for all $x \in X$ and $\left\|f_{1}\right\|=\left\|f_{2}\right\|=\alpha$.

Then $\left(f_{1}-f_{1} f_{2}\right)(\mathrm{x})=\left(f_{1}(\mathrm{x})-f_{1}(x) f_{2}(x)\right) \geq 0$ for all $x \in X$.

Then $f_{1} f_{2} \leq f_{1}$. In the same way we obtain $f_{1} f_{2} \leq f_{2}$.

To show that condition (3) satisfies .

Let $\mathrm{k} f_{\lambda} \cdot\left(1+k f_{\lambda}\right)^{-1} \epsilon C_{0}^{+}(X)$ and $\left\|\mathrm{k} f_{\lambda} \cdot\left(1+k f_{\lambda}\right)^{-1}\right\| \leq 1$,

$0 \leq \mathrm{k} f_{\lambda}(x) \cdot\left(1+k f_{\lambda}(x)\right)^{-1} \leq \frac{1}{k}$ for each,$k \in \mathbb{N}$. 
Therefor

$$
\begin{aligned}
& \left\|f-f_{\lambda}\left(\mathrm{k} f_{\lambda} \cdot\left(1+k f_{\lambda}\right)^{-1}\right)\right\|=\left\|\frac{f_{\lambda}\left(1+k f_{\lambda}\right)-k f_{\lambda}^{2}}{1+k f_{\lambda}}\right\|=\left\|\frac{f_{\lambda}+k f_{\lambda}^{2}-k f_{\lambda}^{2}}{1+k f_{\lambda}}\right\|=\left\|\frac{f_{\lambda}}{1+k f_{\lambda}}\right\| \\
& \left.=\left\|f_{\lambda}\left(1+k f_{\lambda}\right)^{-1}\right\|=\sup _{f_{\lambda}}(x)\left(1+k f_{\lambda}(x)\right)^{-1}: x \in X\right\} \leq \frac{1}{k} .
\end{aligned}
$$

It follows that $\lim _{n} f_{\lambda}\left(\mathrm{k} f_{\lambda} \cdot\left(1+k f_{\lambda}\right)^{-1}=\lim _{n}\left(\mathrm{k} f_{\lambda} \cdot\left(1+k f_{\lambda}\right)^{-1} \cdot f_{\lambda}\right.\right.$.

there is a net $\left\{f_{\lambda}\right\}_{\lambda \in \Lambda}$, such that $f_{\lambda} a \rightarrow$ a satisfying for all $a \in A$.

Now to prove the sufficient part

i.e. Proved that $f_{\lambda} \mathrm{a} \rightarrow \mathrm{a}$ satisfying for all $\mathrm{a} \in A$.

$\sigma$ be the normality constant and $\Lambda$ be the set of all positive elements $\lambda$ with $\|\lambda\|=\alpha$ and let $f_{\lambda}=\lambda$ such that $\left\{f_{\lambda}\right\}_{\lambda \in \Lambda}$ be a net we first show that $f_{\lambda} \mathrm{a} \rightarrow \mathrm{a}$ for all $\mathrm{a} \in A$ and $a \geq 0$ let $a \geq 0$.If $a=0$ the equation directly, suppose $a \neq 0$.We will assume that $\|a\|=\alpha$, let $0 \leq \varepsilon \leq 1$ from (3) there is $b \geq 0$ and $\|b\| \leq 1$ such that $\|a-b a\|<\epsilon$ let $\lambda_{1}=\|b\|^{-1} b$ and suppose that $\lambda \epsilon \Lambda$ such that $\lambda \geq \lambda_{1}$ obtain $\lambda \geq\|b\|^{-1} b \geq b$ so $(\lambda-b) a \epsilon C$ and thus $a-f_{\lambda} a=a-\lambda a \leq a-b a$.

From (2) obtain $f_{\lambda} \mathrm{a} \leq a$, so $a-f_{\lambda} \mathrm{a} \geq 0$

this shown $\left\|a-f_{\lambda} \mathrm{a}\right\| \leq \sigma\|\mathrm{a}-\mathrm{b} a\|<\sigma \varepsilon$ forall $\lambda \in \Lambda, \lambda \geq \lambda_{1}$.

then with arbitrary $\varepsilon$ obtain $f_{\lambda} \mathrm{a} \rightarrow \mathrm{a}$ for all $\mathrm{a} \in A$ with $a \geq 0$.

Now when $\mathrm{a} \in A$.If $a=0$ the equation directly. Suppose $a \neq 0$, from (1) there exist $b \geq 0$ such that $b \geq-a\|a\|^{-1}, a\|a\|^{-1}$.

Let $a=a_{1}-a_{2}$ such that $a_{1}=\frac{1}{2}(b\|a\|+\mathrm{a})$ and $a_{2}=\frac{1}{2}(b\|a\|-\mathrm{a})$.therefore $a_{1}, a_{2} \geq 0$. Since $f_{\lambda} \mathrm{a} \rightarrow \mathrm{a}$ for all $\mathrm{a} \in A$ with $a \geq 0$.

Then $\lim _{\lambda \epsilon \wedge} f_{\lambda} a_{1}=a_{1}$ andlim ${ }_{\lambda \in \wedge} f_{\lambda} a_{2}=a_{2}$.

Thus $\lim _{\lambda \epsilon \Lambda} f_{\lambda} a=\lim _{\lambda \epsilon \Lambda} f_{\lambda}\left(a_{1}-a_{2}\right)=\lim _{\lambda \epsilon \Lambda} f_{\lambda} a_{1}-\lim _{\lambda \epsilon \Lambda} f_{\lambda} a_{2}=a_{1}-a_{2}=a$.

Then we obtain $f_{\lambda} \mathrm{a} \rightarrow \mathrm{a}$ for all $\mathrm{a} \in A$

\section{References}

[1] D.Holland "Banach Algebra Notes", OregonState University .American, February 2015.

[2] D.W. Robinson and S. Yammer, "Addition of an identity to an ordered Banach Space", J. Austral. Math. Soc. 35 (1983), $200-$ 210.

[3] H.Raubenheimer and S. Rode "Cone in Banach Algebra", M.Sc.thesis, University Of the Orange Free State, Indag.Mathem .N.S 7(4).489.502, 1996.

[4] J.B. Conway," A course in Functional Analysis", NewYork, 1990.

[5] J.M. Erdman ", Lecture Notes on Operator Algebra", Portland state University, Ph.D. thesis,Portland State University,2011.

[6] R. de Jong," Ordered Banach Algebras" Ms. Thesis, Leiden University, 2010. 